\title{
LIPOSSOMAS: PROPRIEDADES FÍSICO-QUÍMICAS E FARMACOLÓGICAS, APLICAÇÕES NA QUIMIOTERAPIA À BASE DE ANTIMÔNIO
}

\section{Frédéric Frézard* e Dante A. Schettini}

Departamento de Fisiologia e Biofísica, Instituto de Ciências Biológicas, Universidade Federal de Minas Gerais, Av. Antônio Carlos, 6627, 31270-901 Belo Horizonte - MG

Olguita G. F. Rocha

Laboratório de Análise de Traces Metálicos, Fundação Centro Tecnológico de Minas Gerais, Belo Horizonte - MG Cynthia Demicheli

Departamento de Química, Instituto de Ciências Exatas, Universidade Federal de Minas Gerais, Av. Antônio Carlos, 6627, 31270-901 Belo Horizonte - MG

Recebido em 24/5/04; aceito em 14/9/04; publicado na web em 17/2/05

\begin{abstract}
LIPOSOMES: PHYSICOCHEMICAL AND PHARMACOLOGICAL PROPERTIES, APPLICATIONS IN ANTIMONY-BASED CHEMOTHERAPY. The use of organoantimonial complexes in the therapeutic of leishmaniasis and schistosomiasis has been limited mainly by the need for daily parenteral administration, their adverse side-effects and the appearance of drug resistance. Liposome encapsulation has been so far the most effective means to improve the efficacy of pentavalent antimonials against visceral leishmaniasis. Pharmacologically- and pharmaceutically-acceptable liposomal compositions are still being investigated through manipulation of preparation method, lipid composition and vesicle size. Recently, the encapsulation of a trivalent antimonial within "stealth" liposomes was found to reduce its acute toxicity and effectively deliver this compound to the parasite in experimental schistosomiasis.
\end{abstract}

Keywords: liposomes; antimony; chemotherapy.

\section{INTRODUÇÃO}

No início do século passado, Gaspar Vianna, pesquisador pioneiro em doença de Chagas e leishmaniose, relatou a eficácia do complexo de antimônio trivalente (Sb(III)), tártaro emético, no tratamento da leishmaniose muco-cutânea ${ }^{1}$. Da mesma forma, o tártaro emético foi o primeiro medicamento empregado com êxito no tratamento da esquistossomose ${ }^{2,3}$. Entretanto, o uso clínico deste composto foi interrompido, por causa de seus severos efeitos colaterais e da descoberta de novos fármacos menos tóxicos.

A partir da década de 1940, complexos de antimônio pentavalente $(\mathrm{Sb}(\mathrm{V}))$ começaram a ser utilizados na terapêutica das leishmanioses ${ }^{4}$. Os principais antimoniais atualmente em uso são complexos de $\mathrm{Sb}(\mathrm{V})$ com o N-metil-glucamina (antimoniato de meglumina) e com o gluconato de sódio (estibogluconato de sódio). Até hoje, nem a estrutura desses compostos, nem seu mecanismo de ação foram completamente elucidados. Foi sugerido que o $\mathrm{Sb}(\mathrm{V})$ seria uma pró-droga, sendo reduzido no organismo hospedeiro a $\mathrm{Sb}(\mathrm{III})$ que seria a forma ativa e tóxica ${ }^{5}$. Recentemente, foi mostrado que os tióis podem estar envolvidos nesse processo de redução ${ }^{6}$. Embora os antimoniais pentavalentes continuem sendo os medicamentos de primeira escolha no tratamento de todas as formas de leishmanioses, o seu uso clínico apresenta várias limitações. Esses compostos devem ser administrados por via parenteral (injeção intravenosa ou intramuscular), diariamente, num período de 20-40 dias. Nesse contexto, efeitos colaterais são freqüentes ${ }^{4}$. $\mathrm{O}$ aparecimento de resistência representa um outro problema sério no tratamento das leishmanioses ${ }^{4}$ e um risco potencial na terapêutica da esquistossomose ${ }^{7}$. Em face dessas limitações, a Organização Mundial da Saúde recomenda, com

*e-mail: frezard@mono.icb.ufmg.br incentivo inclusive a outras entidades afins como a TDR ("Special Programme for Research and Training in Tropical Diseases"), a pesquisa de novos medicamentos ${ }^{8}$.

Essencialmente duas estratégias diferentes estão atualmente disponíveis para o desenvolvimento de novos medicamentos. Uma estratégia envolve o planejamento/síntese de novas substâncias ativas ou de fármacos já conhecidos com modificações químicas; a outra envolve a associação reversível de fármacos já em uso a um sistema transportador, visando direcionar o fármaco para a célula alvo e evitar os locais indesejáveis onde o fármaco exerce toxicidade. Esta última estratégia, além de prolongar a validade de proteção por patente no uso do fármaco, oferece um ganho de tempo na fase de desenvolvimento do produto porque usa um fármaco já caracterizado do ponto de vista farmacológico. Entre os sistemas transportadores de medicamentos atualmente disponíveis, os lipossomas ocupam uma posição de destaque, tanto para terapia das leishmanioses quanto da esquistossomose.

Como ilustrado na Figura 1, os lipossomas são vesículas esféricas, constituídas de uma ou várias bicamadas concêntricas de lipídeos, que isolam um ou vários compartimentos aquosos internos do meio externo ${ }^{9}$. Uma grande vantagem dos lipossomas, com relação a outros sistemas transportadores de medicamento, é a sua elevada biocompatibilidade, especialmente quando estes são formados de lipídeos pertencentes às famílias de lipídeos naturais ${ }^{9}$. Além disso, são sistemas altamente versáteis, cujo tamanho, lamelaridade, superfície, composição lipídica, volume e composição do meio aquoso interno podem ser manipulados em função dos requisitos farmacêuticos e farmacológicos.

A capacidade dessas vesículas artificiais oferecerem barreiras para a difusão de solutos foi demonstrada pela primeira vez por Bangham, em $1965^{10}$. O primeiro resultado espectacular na área biomédica foi obtido, na década de 1970, com os antimoniais. Um aumento de até 


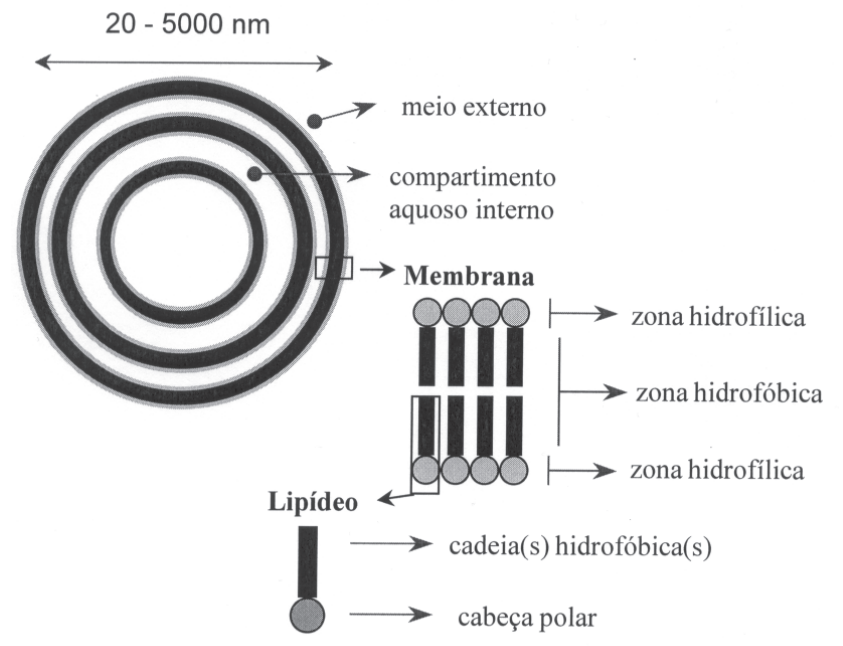

Figura 1. Características estruturais dos lipossomas

700 vezes na atividade leishmanicida desses compostos foi relatado após administração na forma encapsulada em lipossomas ${ }^{11}$.

Apesar da necessidade de aprimorar a quimioterapia antimonial atual e dos resultados extremamente promissores obtidos com os lipossomas em modelos experimentais de leishmaniose visceral, até hoje, nenhuma composição farmacêutica associando lipossomas e antimoniais chegou a ser comercializada. Este fato pode ser atribuído, pelo menos em parte, às dificuldades tecnológicas inerentes à obtenção de formulação estável de compostos hidrossolúveis encapsulado em lipossomas.

Nesse artigo, apresentaremos propriedades básicas dos lipossomas, de interesse para o desenvolvimento de fármaco na forma encapsulada em lipossomas. Mostraremos os aspectos farmacológicos mais relevantes no uso dos lipossomas "convencionais" para a terapêutica das leishmanioses. Apresentaremos os progressos tecnoló- gicos alcançados, nos últimos anos, na preparação dos antimoniais pentavalentes na forma encapsulada em lipossomas. Por outro lado, mostraremos que lipossomas "furtivos" contendo antimoniais trivalentes são promissores para o tratamento da esquistossomose mansoni.

\section{PROPRIEDADES BÁSICAS DOS LIPOSSOMAS}

\section{Composição e propriedades físico-químicas}

Os lipossomas, classicamente, são preparados a partir do glicerofosfolipídeo, fosfatidilcolina. De uma forma mais geral, lipossomas podem ser obtidos a partir de qualquer substância anfifílica formadora de fase lamelar'. A Tabela 1 apresenta exemplos de lipídeos utilizados na preparação de lipossomas. Tipicamente, o diâmetro médio dos lipossomas varia de 20 a $5000 \mathrm{~nm}$. Quando formada de lipídeo de temperatura de transição de fase $\left(T_{\mathrm{t}}\right)$ menor que a temperatura do meio estudado, a membrana dos lipossomas se encontra na fase cristal-líquido ou "fluida" e os lipídeos e suas cadeias de hidrocarboneto têm grande liberdade de movimento (Figura 2). Exemplos desse comportamento podem ser vistos com lipossomas formados de dimiristoilfosfatidilcolina $\left(T_{\mathrm{t}}=23^{\circ} \mathrm{C}\right)$ ou de dioleilfosfatidilcolina $\left(T_{\mathrm{t}}\right.$ $<0{ }^{\circ} \mathrm{C}$ ). Quando formada de lipídeo de $T_{\mathrm{t}}$ maior que a temperatura do meio estudado, a membrana dos lipossomas se encontra na fase gel ou "rígida" e os lipídeos têm movimento restrito e suas cadeias de hidrocarboneto apresentam conformação "toda-trans" (Figura 2). Vesículas formadas de diestearoilfosfatidilcolina $\left(T_{t}=55^{\circ} \mathrm{C}\right)$ representam um exemplo deste tipo de lipossomas. Um componente lipídico importante, que entra muitas vezes na composição dos lipossomas, é o colesterol. Este aumenta a rigidez das membranas no estado "cristallíquido" e reduz a rigidez e os defeitos estruturais das membranas no estado "gel" (Figura 2). Lipídeos apresentando carga efetiva negativa ou positiva podem também ser incluídos na composição da membrana o que pode influenciar a taxa de incorporação de substâncias, impedir a agregação/fusão das vesículas lipídicas e modular o seu destino no organismo.

Tabela 1. Exemplos de lipídeos formadores de bicamada, utilizados na preparação de fámacos na forma encapsulada em lipossomas

\begin{tabular}{|c|c|c|}
\hline $\begin{array}{l}\text { Família de lipídeos } \\
\text { Fórmula estrutural }\end{array}$ & $\begin{array}{l}\text { R (cadeia hidrofóbica) } \\
\text { (nome) }\end{array}$ & $\begin{array}{l}\text { Abreviação } \\
\left(T_{\mathrm{t}}\right)\end{array}$ \\
\hline \multirow{5}{*}{$\stackrel{\mathrm{R}-\mathrm{O}}{\mathrm{R}-\mathrm{O}} \underset{\substack{\mathrm{O}-\mathrm{P}-\mathrm{O}-\mathrm{CH}_{2}-\mathrm{CH}_{2}-\mathrm{N} \\
\mathrm{O}^{-}}}{\mathrm{O}}=$} & & \\
\hline & $\begin{array}{c}\mathrm{CH}_{3}-\left(\mathrm{CH}_{2}\right)_{7}-\mathrm{CH}=\mathrm{CH}-\left(\mathrm{CH}_{2}\right)_{7}-\mathrm{C}(\mathrm{O})- \\
\text { (oleil) }\end{array}$ & $\begin{array}{l}\text { DOPC } \\
\left(<0^{\circ} \mathrm{C}\right)\end{array}$ \\
\hline & $\begin{array}{l}\mathrm{CH}_{3}-(\mathrm{CH} 2)_{12}-\mathrm{C}(\mathrm{O})- \\
\text { (miristoil) }\end{array}$ & $\begin{array}{c}\text { DMPC } \\
\left(+23{ }^{\circ} \mathrm{C}\right)\end{array}$ \\
\hline & $\begin{array}{l}\mathrm{CH}_{3}-(\mathrm{CH} 2)_{14}-\mathrm{C}(\mathrm{O})- \\
\text { (palmitoil) }\end{array}$ & $\begin{array}{c}\mathrm{DPPC} \\
\left(+42^{\circ} \mathrm{C}\right)\end{array}$ \\
\hline & $\begin{array}{l}\mathrm{CH}_{3}-(\mathrm{CH} 2)_{16}-\mathrm{C}(\mathrm{O})- \\
\text { (estearoil) }\end{array}$ & $\begin{array}{c}\text { DSPC } \\
\left(+55^{\circ} \mathrm{C}\right)\end{array}$ \\
\hline \multicolumn{3}{|l|}{ Fosfatidilglicerol } \\
\hline \multirow{2}{*}{ 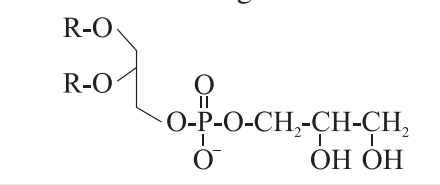 } & $\begin{array}{l}\mathrm{CH}_{3}-(\mathrm{CH} 2)_{12}-\mathrm{C}(\mathrm{O})- \\
\text { (miristoil) }\end{array}$ & $\begin{array}{l}\text { DMPG } \\
\left(+13^{\circ} \mathrm{C}\right)\end{array}$ \\
\hline & $\begin{array}{l}\mathrm{CH}_{3}-(\mathrm{CH} 2)_{14}-\mathrm{C}(\mathrm{O})- \\
\text { (palmitoil) }\end{array}$ & $\begin{array}{c}\mathrm{DPPG} \\
\left(+35^{\circ} \mathrm{C}\right)\end{array}$ \\
\hline \multicolumn{3}{|l|}{$\begin{array}{l}\text { Alquil polioxietileno éter (exemplo } \\
\text { de surfactante não iônico) }\end{array}$} \\
\hline $\mathrm{R}-\left(\mathrm{O}-\mathrm{CH}_{2}-\mathrm{CH}_{2}\right)_{6}-\mathrm{CH}_{2}-\mathrm{CH}_{2} \mathrm{OH}$ & $\begin{array}{l}\mathrm{CH}_{3}-(\mathrm{CH} 2)_{15}- \\
\quad(\text { cetil })\end{array}$ & $\begin{array}{l}\mathrm{C}_{16} \mathrm{EO}_{7} \\
\left(+35^{\circ} \mathrm{C}\right)\end{array}$ \\
\hline
\end{tabular}




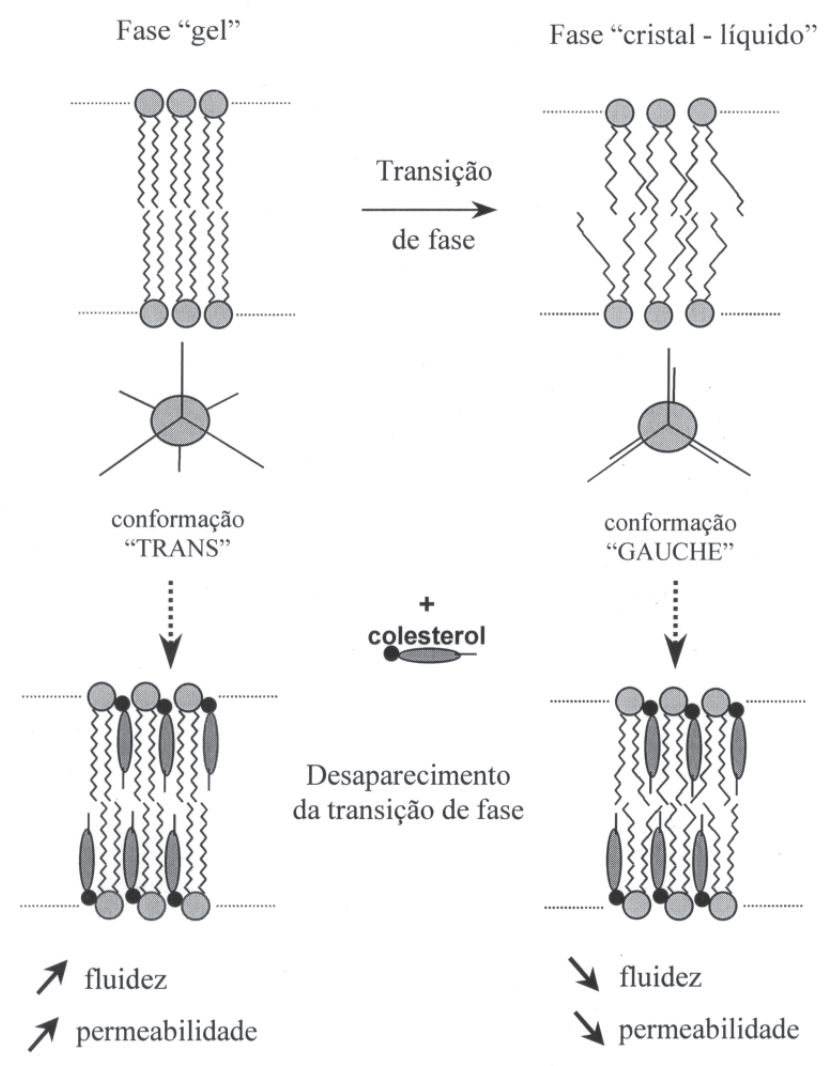

Figura 2. Comportamento de fase das membranas lipídicas e efeito do colesterol. Uma membrana, composta por um determinado tipo de lipídeo e caracterizada por uma determinada $\mathrm{T}_{p}$ encontra-se na fase gel (ligações C-C na conformação "toda-trans") abaixo da $\mathrm{T}_{t}$ e na fase cristal-líquido (ligações C-C na conformação "trans" ou "gauche") acima da $\mathrm{T}_{i}$. O colesterol "apaga" a transição de fase da membrana e mantém a membrana em estado de fluidez intermediária. O colesterol aumenta a permeabilidade da membrana na fase gel e a reduz na fase fluida

\section{Capacidade de encapsulação de substância}

Substâncias farmacologicamente ativas podem ser incorporadas seja no compartimento aquoso interno (substâncias hidrossolúveis), seja nas membranas dos lipossomas (substâncias lipofílicas ou anfifílicas).

A taxa de encapsulação de uma substância em lipossomas e a relação substância encapsulada/lipídeo são dois parâmetros importantes que devem ser considerados na escolha do método de preparação, sobretudo quando se procura desenvolver uma composição farmacêutica. Esses parâmetros podem ser otimizados através da escolha do método de encapsulação e da manipulação da composição lipídica da membrana. A taxa de encapsulação deverá ser maximizada, pois é inversamente relacionada à quantidade de substância não encapsulada que é perdida na maioria das vezes. A relação fármaco/ lipídeo deverá também ser maximizada, visto que determina a quantidade de lipídeo a ser administrada ao paciente. Assim, quanto menor for a quantidade de lipídeo veiculada, menor serão os riscos de efeitos colaterais associados aos mesmos.

Teoricamente, taxas de encapsulação próximas de $100 \%$ podem ser obtidas com substâncias lipofílicas incorporadas na membrana dos lipossomas. É o caso da anfotericina B que, no produto farmacêutico AmBisome, é associada a lipossomas pequenos (45-80 nm de diâmetro) formados de fosfatidilcolina hidrogenada, colesterol e de diestearoilfosfatidilglicerol (relação molar 2:1:0,8 e relação fármaco/lipídeo = 1/10). Vale ressaltar que a capacidade de substâncias lipofílicas serem incorporadas na membrana de lipossomas não é uma regra geral; no processo de encapsulação, deve ser verificado se a substância está efetivamente incorporada na membrana e não simplesmente adsorvida na sua superfície ou auto-associada na forma de microcristais em suspensão com os lipossomas. No caso das substâncias hidrossolúveis, como os antimoniais pentavalentes, é teoricamente impossível atingir níveis de encapsulação de 100\%, por causa da necessária co-existência dos compartimentos aquosos interno e externo.

\section{Fatores determinantes na cinética de liberação da substância encapsulada}

Lipossomas, contendo uma substância encapsulada no seu compartimento aquoso interno, não se encontram em equilíbrio termodinâmico, quando são resuspendidos em meio aquoso sem a substância. A evolução do estado desse sistema levará necessariamente à liberação da substância para o meio externo até que se atinja um novo equilíbrio.

No caso das substâncias incorporadas nas membranas dos lipossomas, a quantidade de substância liberada dependerá do coeficiente de partição da substância entre a membrana e a fase aquosa, assim como dos componentes desta fase.

No caso das substâncias incorporadas no compartimento aquoso, a criação de um gradiente de concentração da substância através da membrana faz com que esta atue como barreira, limitando a velocidade de difusão das substâncias para o meio externo. Este sistema evoluirá espontaneamente para o equilíbrio termodinâmico, ou seja, para a igualdade das concentrações interna e externa de substância.

Nesse contexto, torna-se importante poder prever a cinética de liberação da substância encapsulada e conhecer os parâmetros físico-químicos que vão influenciá-la. Descreveremos abaixo um modelo simples que nos permite ter acesso a essas informações.

O fluxo de substância através da membrana $\left(J_{\mathrm{S}}=\right.$ número de mols de substância que atravessa a membrana por unidade de tempo e unidade de área da membrana) dependerá da concentração da substância no compartimento aquoso interno $\left(C_{\mathrm{i}}\right)$ e do coeficiente de permeabilidade da substância através da membrana $\left(P_{\mathrm{S}}\right)$, de acordo com a lei de difusão simples:

$J_{\mathrm{S}}=P_{\mathrm{S}} C_{\mathrm{i}}$

No caso de lipossomas unilamelares de distribuição de tamanho homogêneo, de volume e de área internas $V_{\mathrm{i}}$ e $S_{\mathrm{i}}$, respectivamente, o fluxo $J_{\mathrm{S}}$ tem a seguinte expressão:

$J_{\mathrm{S}}=-\left(1 / S_{\mathrm{i}}\right)(\mathrm{dn} / \mathrm{dt})$

onde n é o número de mols de substância no compartimento interno.

Podemos deduzir:

$J_{\mathrm{S}}=-\left(V_{\mathrm{i}} / S_{\mathrm{i}}\right)\left(\mathrm{d} C_{\mathrm{i}} / \mathrm{dt}\right)$

onde $C_{\mathrm{i}}$ é a concentração de substância no compartimento interno.

Podemos também escrever:

$J_{\mathrm{S}}=-\left(R_{\mathrm{i}} / 3\right)\left(\mathrm{d} C_{\mathrm{i}} / \mathrm{dt}\right)$

onde $R_{\mathrm{i}}$ é o raio interno médio dos lipossomas.

A partir das Equações 1 e 4, podemos deduzir a seguinte relação:

$\mathrm{d} C_{\mathrm{i}} / C_{\mathrm{i}}=-\left(3 P_{\mathrm{s}} / R_{\mathrm{i}}\right) \mathrm{dt}$ 
que, após integração, leva à seguinte relação:

$C_{\mathrm{i}}=C_{\mathrm{io}} \exp \left(-3 P_{\mathrm{s}} \mathrm{t} / R_{\mathrm{i}}\right)$

onde $C_{\text {io }}$ é a concentração interna de substância no tempo zero.

A Equação 6 mostra que a cinética de liberação da substância, por mecanismo de difusão simples, a partir de lipossomas de distribuição de tamanho homogêneo, é monoexponencial e determinada pelo coeficiente de permeabilidade da substância e pelo tamanho dos lipossomas. Quanto menor for o coeficiente de permeabilidade da substância ou quanto maior for o tamanho dos lipossomas, mais lenta será a liberação da substância dos lipossomas.

Vale ressaltar que o coeficiente de permeabilidade, $P_{\mathrm{s}}$, depende do coeficiente de partição da substância entre a membrana e a fase aquosa $(k=$ concentração na membrana/concentração na fase aquosa), da constante de difusão da substância na membrana $\left(D_{\mathrm{S}}\right)$ e da espessura da membrana $(\Delta \mathrm{x})$, de acordo com a expressão:

$P_{\mathrm{S}}=k D_{\mathrm{s}} / \Delta \mathrm{x}$

No caso das moléculas esféricas, $D_{\mathrm{S}}$ dependerá do raio molecular $\left(r_{\mathrm{m}}\right)$ e da viscosidade da membrana $(\eta)$, de acordo com a relação de Stockes-Einstein:

$D_{\mathrm{S}}=R T / 6 \pi \mathrm{N} \eta r_{\mathrm{m}}$

onde $\mathrm{N}$ é o número de Avogadro, $R$ a constante dos gases perfeitos e $T$ a temperatura absoluta.

Podemos deduzir que substâncias de grande tamanho $\left(D_{\mathrm{S}}\right.$ pequeno) e elevada polaridade ( $k$ pequeno) serão retidas com maior eficiência no compartimento aquoso interno dos lipossomas que as substâncias de pequeno tamanho e de baixa polaridade, respectivamente. Além disso, como a constante de difusão da substância é inversamente proporcional à viscosidade da membrana, podemos prever que membranas na fase gel reterão a substância encapsulada com maior eficiência que membranas na fase cristal-líquido. Por exemplo, membranas constituídas de diestearoilfosfatidilcolina mostraram-se, a $37{ }^{\circ} \mathrm{C}$, aproximadamente 6 vezes menos permeáveis à carboxifluoresceína que membranas constituídas de dimiristoilfosfatidilcolina ${ }^{12}$.

A pressão osmótica do meio interno dos lipossomas é um outro fator importante que vai influenciar a liberação do princípio ativo encapsulado. Como as bicamadas lipídicas são muito permeáveis à água, a existência de uma diferença de pressão osmótica entre os meios interno e externo induzirá um movimento de água por osmose através da membrana. Portanto, deve-se evitar preparar lipossomas com pressão osmótica interna superior à externa, pois isto provoca a entrada de água nos lipossomas, podendo induzir um choque osmótico com conseqüente liberação da substância encapsulada.

O armazenamento dos lipossomas na forma de suspensão aquosa é muitas vezes incompatível com a estabilidade requerida para os produtos farmacêuticos (de pelo menos um ano), mesmo se a substância encapsulada apresenta um baixo coeficiente de permeabilidade ou se a preparação é mantida na forma de sedimento com pequeno volume externo. Assim, outras formas de armazenamento devem ser procuradas para os produtos farmacêuticos à base de lipossomas. $\mathrm{O}$ armazenamento na forma liofilizada representa uma alternativa viável, entretanto, deve ser incluído na formulação um agente crioprotetor para evitar a fusão das membranas desidratadas e o conseqüente vazamento da substância encapsulada no momento da reidratação ${ }^{13}$.

Quando administrados em organismos vivos, os lipossomas interagem, no primeiro momento, com os componentes dos fluidos biológicos, o que pode alterar a permeabilidade de sua membrana e a velocidade de liberação da substância encapsulada. A Figura 3 ilustra as interações dos lipossomas com dois fluidos biológicos importantes: os sais biliares e o soro. Após administração dos lipossomas por via oral, os sais biliares se incorporam na sua membrana, tendendo induzir a transição da fase lamelar para a fase micelar. Nestas condições, os lipossomas "fluidos", que apresentam membrana na fase cristal-líquido, serão desestabilizados. Apenas lipossomas com membrana na fase gel parecem resistir parcialmente à ação dos sais biliares ${ }^{14}$. Após administração dos lipossomas por via endovenosa, estes interagem com as lipoproteínas plasmáticas. Lipídeos podem ser transferidos dos lipossomas para as lipoproteínas, enquanto componentes das lipoproteínas (como a apolipoproteína) podem ser incorporados na membrana dos lipossomas. Nessas condições, lipossomas "fluidos" manterão sua integridade apenas se estes contiverem colesterol na proporção de pelo menos $30 \%$ do total de lipídeos. Por outro lado, lipossomas com membrana na fase gel ficam mais refratários às trocas com as lipoproteínas e, portanto, mantém sua elevada capacidade de retenção.

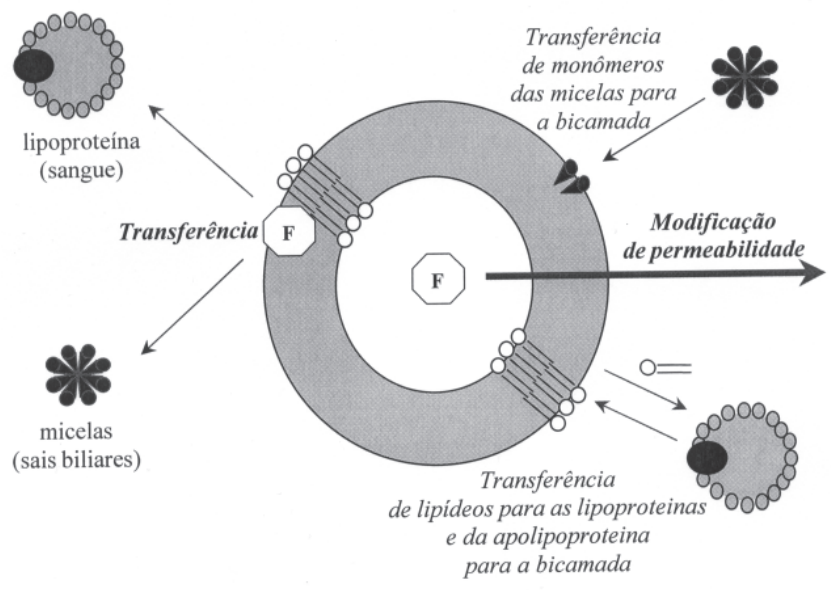

Figura 3. Efeito das lipoproteínas plasmáticas e dos sais biliares na retenção de fármaco encapsulado pelos lipossomas. Esses componentes biológicos aumentam a permeabilidade da membrana aos fármacos hidrossolúveis $e$ oferecem um meio para a transferência dos fármacos lipossolúveis

\section{Interação lipossomas/células: influência na biodisponibilidade da substância encapsulada}

Como visto anteriormente, os lipossomas são capazes de reterem princípios ativos encapsulados e, portanto, de protegê-los in vivo de uma eliminação ou degradação rápida. Suas propriedades de liberação lenta levam à redução da concentração de princípio ativo na forma livre, e à prolongação de sua presença no organismo. Esse aumento na biodisponibilidade do princípio ativo pode levar à potenciação da sua ação biológica, assim como à redução dos seus efeitos colaterais.

Enquanto a liberação da substância encapsulada em condição de armazenamento é passiva e espontânea, sua liberação in vivo a partir de lipossomas estáveis será mediada essencialmente por células com atividade endocitária. Nesse contexto, a manipulação das características de superfície dos lipossomas representa um dos meios atualmente disponíveis para controlar sua interação com as células e a velocidade de liberação da substância nos organismos vivos.

Como ilustrado na Figura 4, os lipossomas convencionais (formados de fosfatidilcolina ou de surfactantes não-iônicos e de colesterol), quando administrados por via endovenosa, são natural- 
mente capturados pelos macrófagos do sistema mononuclear fagocitário, principalmente do fígado, do baço e da medula óssea. Portanto, a administração de fármacos na forma encapsulada nesses lipossomas resulta no aumento de sua concentração nesses órgãos, assim como na redução da concentração em órgãos apresentando capilares contínuos. A captura celular dos lipossomas é favorecida pelo processo de opsonização, que ocorre assim que os lipossomas entram em contato com os componentes do sangue. Depois da fagocitose, os lipossomas são degradados pelas fosfolipases lisossomais e a substância é liberada nos fagolisossomas, podendo se difundir para o citossol ou ser excretada para o meio extracelular. A velocidade de liberação do princípio ativo pode ser controlada pela manipulação tanto da composição da membrana (influenciando a velocidade de degradação dos lipossomas), quanto do tamanho dos lipossomas (influenciando a eficiência de captura pelos macrófagos). Assim, lipossomas pequenos serão capturados com menor eficiência que lipossomas grandes, permanecerão mais tempo na circulação sanguínea e apresentarão uma liberação mais prolongada.

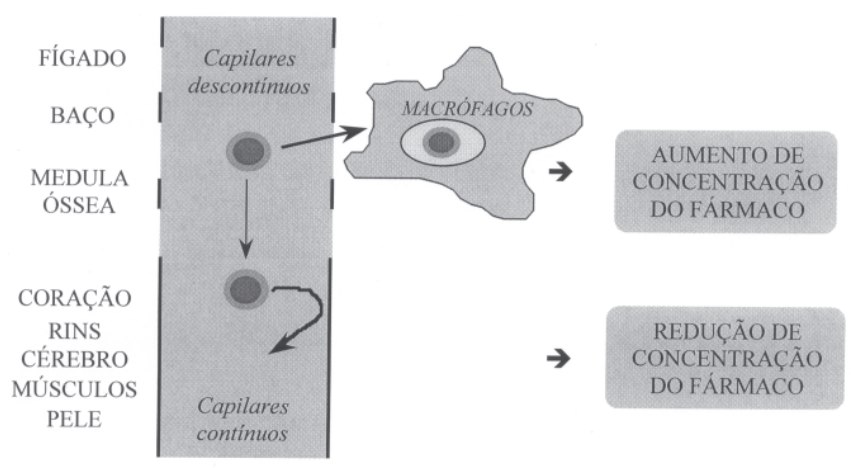

Figura 4. Destino dos lipossomas convencionais por via endovenosa. Os lipossomas são capturados pelos macrófagos do sistema mononuclear fagocitário e não penetram nos órgãos que apresentam capilares contínuos

A descoberta de que o destino in vivo dos lipossomas poderia ser controlado através da manipulação de suas características de superfície constituiu um dos principais avanços da década de $1990^{15}$. Foi observado que a incorporação na membrana dos lipossomas de lipídeos acoplados a polímeros de etilenoglicol altera suas interações com o ambiente, sendo o efeito mais importante o impedimento da captura pelos macrófagos e a prolongação de sua presença no organismo. Em consequiência, este tipo de lipossomas, chamado de "lipossomas furtivos", promove uma presença prolongada da substância no organismo, além de distribuí-la para outros órgãos, além daqueles do sistema mononuclear fagocitário. Esses lipossomas podem ainda ser direcionados para determinados tipos celulares, via receptores ou ligantes específicos, através do acoplamento de anticorpo ou ligante na extremidade do polímero.

Uma das maiores desvantagens da terapia tópica é a baixa velocidade de penetração dos fármacos através da pele. A barreira difusional da maioria das substâncias é o estrato córneo, que consiste de corneócitos embebidos em uma matriz lipídica. A matriz lipídica do estrato córneo é formada de uma bicamada lipídica composta de colesterol, ácidos graxos e ceramidas. O uso de lipossomas como promotor de absorção cutânea tem sido estudado intensivamente ${ }^{16}$. Numerosos estudos realizados com uma grande variedade de fármacos e lipossomas de diferentes composições mostraram que a aplicação do fármaco na forma encapsulada em lipossomas permite modular sua absorção na epiderme e na derme, assim como sua absorção sistêmica.

\section{APLICAÇÕES DOS LIPOSSOMAS NA QUIMIOTERAPIA ANTIMONIAL}

\section{As doenças alvos}

A esquistossomose é uma doença parasitária crônica causada por espécies do gênero Schistosoma. Estima-se que no Brasil, 8 a 10 milhões de indivíduos estejam infectados e que 30 milhões corram o risco de contrair a doença ${ }^{17}$. A infecção é causada pelas cercárias, liberadas pelo hospedeiro intermediário (molusco), que penetram através da pele do homem quando este entra em contato com a água contaminada ${ }^{17}$. Os parasitas são encontrados nos pulmões, na forma de esquistossômulos, um dia após a penetração das cercárias. Em nove dias, os esquistossômulos vão para o fígado, onde se alimentam e se desenvolvem, alcançando a vida adulta. Os vermes adultos atingem as veias mesentéricas, principalmente a inferior, no caso do S. mansoni.

As leishmanioses são doenças parasitárias causadas por protozoários flagelados pertencentes à Ordem Kinetoplastida, Família Trypanosomatidae e ao Gênero Leishmania. Estas atingem cerca de 12 milhões de pessoas no mundo ${ }^{18}$. No Brasil, dados recentes relatam a ocorrência de cerca de 30.000 novos casos anuais da doença ${ }^{19}$. As leishmanioses são transmitidas aos hospedeiros vertebrados pela picada de um inseto que regurgita o parasita na forma promastigota. Esses parasitas são fagocitados por macrófagos, no interior dos quais estes se transformam em amastigotas. Os amastigotas multiplicamse livremente no compartimento ácido dos fagolisossomas e escapam dos sistemas de defesa do hospedeiro. A Leishmania corresponde a um complexo de várias espécies diferentes que causam vários tipos de manifestações clínicas, que incluem formas cutânea, mucocutânea e visceral. O cão aparece como hospedeiro vertebrado nas formas cutânea e visceral e, particularmente no caso da leishmaniose visceral, tem papel importante como reservatório e fonte de infecção da doença em área endêmica. A leishmaniose visceral apresenta índice de letalidade de $100 \%$ nos casos não tratados clinicamente ${ }^{4}$.

Conhecimentos sobre as características da doença alvo e, principalmente, a localização do parasita, são essenciais na escolha do tipo de lipossoma a ser empregado. Como mostraremos em seguida, a localização da Leishmania nos órgãos do sistema mononuclear fagocitário torna os lipossomas "convencionais" sistemas ideais para concentrar o fármaco especificamente no local de infecção. Por outro lado, a localização do Schistosoma mansoni no sistema porta e seu modo de alimentação com os componentes do sangue sugere que os lipossomas "furtivos" são mais adequados para direcionar o fármaco para o parasita.

\section{Aspectos farmacológicos no uso dos lipossomas no tratamento das leishmanioses}

Em 1977-78, grupos de pesquisa inglês e americano propuseram o uso de lipossomas convencionais contendo drogas leishmanicidas como uma nova abordagem para o tratamento da leishmaniose visceral ${ }^{11,20}$. Esta proposta foi baseada na observação de que antimoniais pentavalentes encapsulados em lipossomas foram 200700 vezes mais eficazes que na forma livre, em modelo experimental de leishmaniose visceral (hamsters ou camundongos infectados por Leishmania donovani). O aumento espectacular de eficácia dessas drogas foi atribuído ao destino natural dos lipossomas quando administrados por via endovenosa: o fato de eles serem captados pelos mesmos órgãos (o fígado, o baço e a medula óssea) e pelas mesmas células (os macrófagos teciduais) nas quais se localiza o parasita. Assim, os lipossomas convencionais apresentam-se como sistema ideal para direcionar drogas leishmanicidas para o local de infecção. 
Estudos de biodistribuição/farmacocinética do antimônio em camundongos e cães mostraram que a forma lipossomal promove níveis de antimônio muito mais elevados e prolongados no fígado e no baço dos animais quando comparado à forma livre ${ }^{21,22}$. Essa diferença de biodistribuição entre as formas livre e encapsulada é ilustrada, na Tabela 2, a partir de dados obtidos em cães.

Tabela 2. Níveis teciduais de antimônio em cães ( $\mu \mathrm{g} / \mathrm{g}$ tecido), $48 \mathrm{~h}$ após injeção intravenosa de antimonial pentavalente, na forma livre e na forma encapsulada, em lipossomas de tipo FDELs

\begin{tabular}{lcccc}
\hline $\begin{array}{l}\text { Forma do antimonial } \\
\text { (dose) }\end{array}$ & Fígado & Baço & Medula óssea & Ref. \\
\hline $\begin{array}{l}\text { Forma livre } \\
(45 \mathrm{mg} \mathrm{Sb} / \mathrm{kg})\end{array}$ & 7 & 2 & 3 & 21 \\
$\begin{array}{l}\text { Forma encapsulada } \\
\text { em lipossomas }\end{array}$ & 44 & 103 & 3 & 22 \\
$(4 \mathrm{mg} \mathrm{Sb} / \mathrm{kg})$ & & & & \\
\hline
\end{tabular}

Vale ressaltar que o tamanho dos lipossomas é um parâmetro importante, pois tem influência tanto na taxa de eliminação do parasita $^{23}$, quanto na concentração de antimônio ao nível da medula ós$\mathrm{sea}^{22}$. Portanto, lipossomas pequenos (diâmetro $<200 \mathrm{~nm}$ ) parecem ter acesso facilitado à medula óssea, o que é de interesse pois devem possibilitar a eliminação do parasita que se encontra nesse tecido.

Uma formulação da anfotericina B à base de lipossomas (AmBisome ${ }^{\circledR}$ ), depois de testes clínicos bem sucedidos, chegou a ser aprovada pela FDA ("Food and Drug Administration") para o tratamento do calazar ${ }^{24}$. Os principais impactos da encapsulação desse fármaco em lipossomas foram o aumento do seu índice terapêutico e o prolongamento de sua ação. Os benefícios clínicos da terapia baseada em lipossomas, quando comparada à quimioterapia convencional, são a redução do número de injeções, da duração da terapia e dos efeitos colaterais. Entretanto, até hoje, o elevado custo comercial do AmBisome limitou o seu uso no Brasil.

O desenvolvimento de formulações tópicas seria altamente desejável para o tratamento da leishmaniose cutânea. Diversos fatores apontam para o desenvolvimento de formulações baseadas em antimoniais encapsulados em lipossomas: a capacidade comprovada dos lipossomas de promoverem a absorção cutânea de fármacos e a eficácia do antimoniato de meglumina intralesional na leishmaniose cutânea ${ }^{25}$.

\section{Aspectos tecnológicos na preparação dos antimoniais pentavalentes na forma encapsulada em lipossomas}

O primeiro método proposto para a encapsulação dos antimoniais pentavalentes em lipossomas foi o método de "hidratação do filme"26. Como ilustrado na Figura 5, este consiste na hidratação de um filme lipídico com uma solução aquosa do antimonial, levando à formação de lipossomas multilamelares chamados de MLVs ("multilamellar vesicles"). As maiores taxas de encapsulação e de retenção foram obtidas com lipossomas constituídos de fosfolipídeo de alta temperatura de transição de fase (dipalmitoilfosfatidilcolina, $T_{\mathrm{t}}=42{ }^{\circ} \mathrm{C}$ ), de colesterol e de fosfolipídeo carregado negativamente.

Um outro método avaliado foi o método de "evaporação em fase reversa" 27 que, como descrito na Figura 6, leva à formação de lipossomas oligolamelares de tipo REVs ("reverse phase evaporation vesicles"). Esse método permite alcançar maiores taxas de encapsulação que o método de hidratação do filme (Tabela 3). Entretanto, lipossomas de tipo REVs se mostraram menos estáveis que aqueles do tipo MLVs, sendo que 25-50\% de antimônio foi liberado

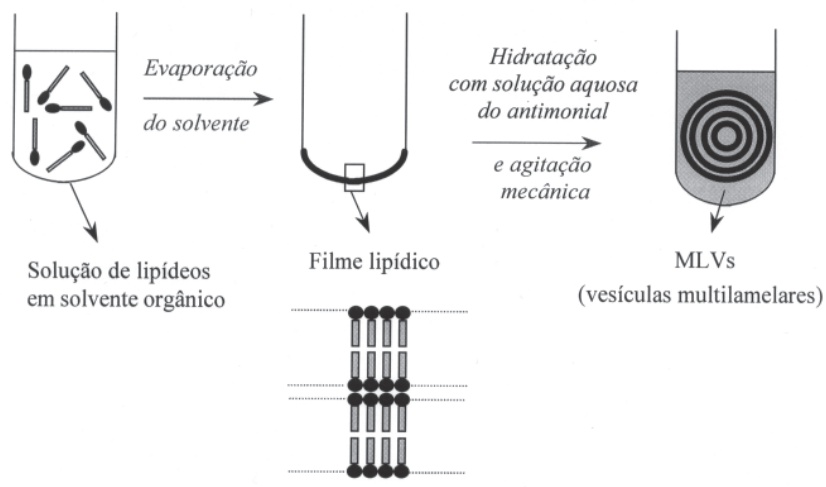

Figura 5. Método de preparação de lipossomas do tipo MLVs, baseado no processo de hidratação do filme lipídico

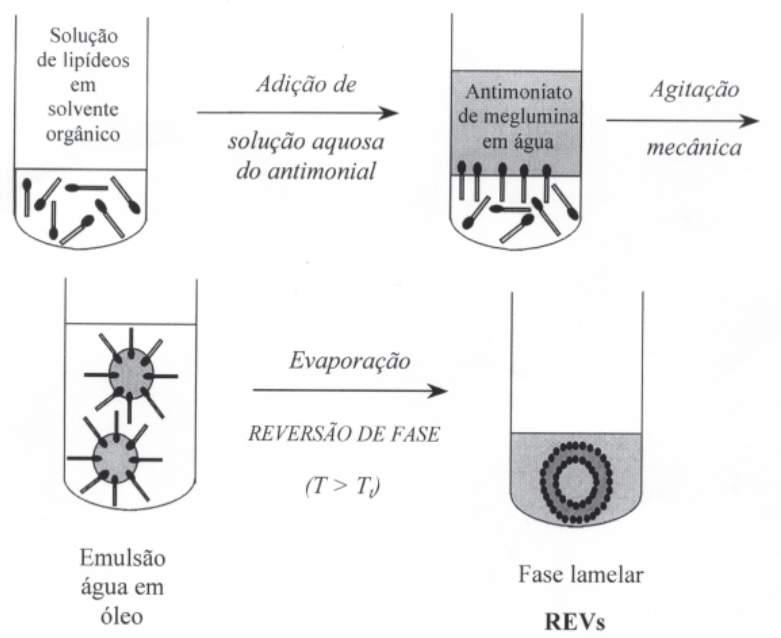

Figura 6. Método de preparação de lipossomas do tipo REVs, baseado no processo de evaporação em fase reversa

da preparação após uma semana de incubação em $25^{\circ} \mathrm{C}$. Por outro lado, o uso de solvente orgânico na preparação desses lipossomas representa um inconveniente, considerando que sempre permanecem resíduos potencialmente tóxicos na formulação final.

Um outro inconveniente dos dois métodos apresentados anteriormente é que as preparações resultantes só podem ser armazenadas na forma de suspensão aquosa, o que limita consideravelmente o seu potencial como produto farmacêutico.

Recentemente, nosso grupo avaliou o método de "desidrataçãoreidratação" desenvolvido por Kirby e Gregoriadis ${ }^{28}$ para a encapsulação do antimoniato de meglumina em lipossomas formados de diestearoilfosfatidilcolina, colesterol e dicetilfosfato ${ }^{29}$. Como mostrado na Figura 7, esse método consiste em misturar uma suspensão de lipossomas pequenos vazios (preparados em água) com uma solução de antimoniato de meglumina e em liofilizar a mistura. A reidratação dessa preparação em condições específicas de temperatura $\left(>T_{\mathrm{t}}\right)$ e de concentração lipídica leva à obtenção de lipossomas com elevada taxa de encapsulação (Tabela 3), chamados de DRVs (“dehydration rehydration vesicles"). Uma alternativa a esse método proposta por nosso grupo ${ }^{29}$ consiste em liofilizar os lipossomas pequenos vazios sem o antimonial e em reidratar o liofilizado, chamado de FDELs ("freeze-dried empty liposomes"), com a solução de antimonial. Esse método apresenta a vantagem de não submeter o antimonial à liofilização, além de permitir uma elevada taxa de encapsulação (Tabela 3). Uma grande vantagem desses dois últimos 
Tabela 3. Características de encapsulação dos antimoniais a partir de lipossomas obtidos por diferentes métodos

\begin{tabular}{cccl}
\hline Tipo de lipossomas & $\begin{array}{c}\text { Taxa de encapsulação } \\
\text { máxima }\end{array}$ & $\begin{array}{c}\text { Relação Sb/lipídeo }(\mathrm{m} / \mathrm{m}) \\
\text { máxima }\end{array}$ & Forma de armazenamento proposta \\
\hline MLVs & $7 \%$ & 0,3 & Suspensão aquosa \\
REVs & $60 \%$ & 0,55 & Suspensão aquosa \\
DRVs & $63 \%$ & 0,37 & Forma liofilizada intermediária \\
FDELs & $42 \%$ & 0,25 & Forma liofilizada intermediária \\
\hline
\end{tabular}

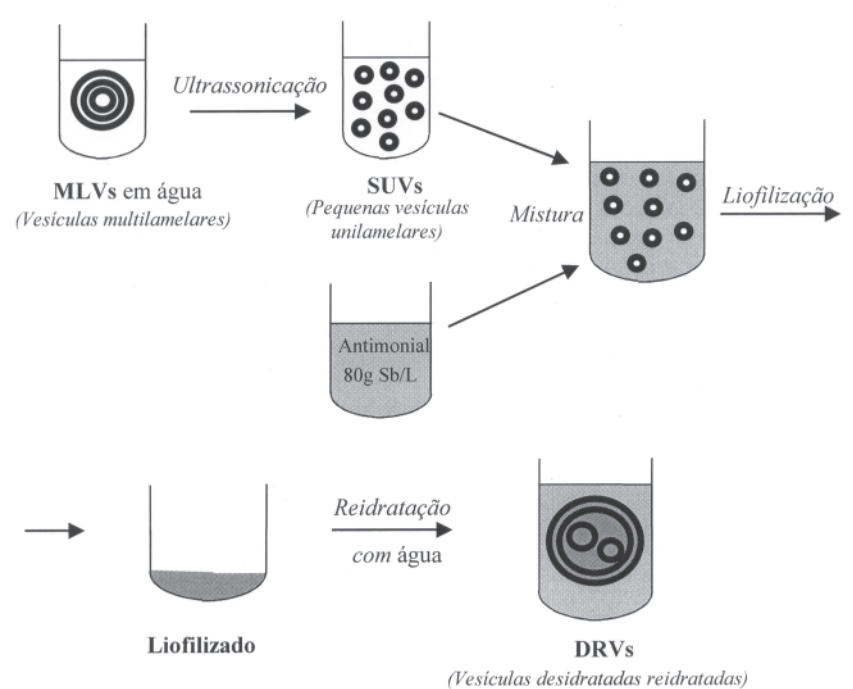

Figura 7. Método de preparação de lipossomas do tipo DRVs, baseado no processo de desidratação - reidratação

métodos com relação aos primeiros é que a preparação pode ser armazenada por tempo prolongado na forma liofilizada intermediária e reidratada um pouco antes do uso. Vale ressaltar que a presença do lipídeo carregado negativamente, dicetilfosfato, é importante para a obtenção de taxa de encapsulação elevada do antimoniato de meglumina. Na sua ausência, taxas de encapsulação inferiores a 15\% são obtidos ${ }^{30}$.

Os diversos tipos de lipossomas obtidos por esses diferentes métodos apresentam diâmetro médio elevado (400 - 5000 nm) e distribuição de tamanho heterogênea. Essa característica representa um problema potencial, pois a administração endovenosa de lipossomas de tamanho grande (>1000 nm) pode levar à obstrução dos capilares pulmonares. Segundo Zadi e Gregoriadis, é possível obter suspensão de lipossomas de tamanho reduzido e mais homogêneo utilizando o método de desidratação-reidratação, mediante ao uso de um açúcar como agente crioprotetor ${ }^{31}$. Como mostrado na Tabela 4, a utilização de sacarose no processo de preparação de lipossomas DRVs e FDELs permite a obtenção de lipossomas de tamanho reduzido. A presença desse açúcar resulta em redução da taxa de encapsulação do antimoniato de meglumina no caso dos lipossomas DRVs, mas não dos lipossomas FDELs. Além disso, como mostrado na Tabela 4, um aumento da velocidade de liberação do antimonial é observado nos lipossomas preparados com sacarose. Esse aumento na velocidade de liberação poderia ser explicado pelo aumento da pressão osmótica do meio interno dos lipossomas, devido à co-encapsulação da sacarose.

\section{Potencial dos lipossomas no tratamento da esquistossomose}

Os primeiros estudos realizados com lipossomas convencionais administrados por via subcutânea evidenciaram a capacidade dos lipossomas de potenciar e prolongar o efeito profilático das drogas esquistossomicidas, oxamniquine ${ }^{32}$, praziquantel $^{33}$ e tártaro emético ${ }^{34}$. O efeito benéfico dos lipossomas foi atribuído às suas propriedades de liberação prolongada, resultando no aumento da biodisponibilidade do princípio ativo. Foi também proposto que os lipossomas promoveriam, através do direcionamento passivo para o fígado, uma maior concentração do fármaco no parasita durante o seu estágio final de diferenciação nesse mesmo tecido.

Recentemente, nosso grupo relatou a preparação de lipossomas de tamanho calibrado (diâmetro médio $=110 \mathrm{~nm}$ ), contendo tártaro emético encapsulado na relação antimônio/lipídeo 0,015 (m/m). Esses lipossomas foram obtidos por filtração de MLVs através de membranas de policarbonato de poro de $100 \mathrm{~nm}$ de diâmetro. Dois tipos diferentes de lipossomas foram avaliados: lipossomas convencionais (LC) formados de diesteraroilfosfatidilcolina e de colesterol e lipossomas furtivos (LF) contendo, além destes lipídeos, o lipídeo diestearoilfosfatidiletanolamina acoplado ao polietilenoglicol 2000. Estes lipossomas mostraram uma elevada estabilidade no soro, com cinética de liberação monoexponencial, de acordo com a lei de difusão simples, e uma meia-vida de liberação de 9 dias.

Estes lipossomas foram avaliados em modelo experimental de esquistossomose mansoni, em estágio tardio da doença ${ }^{35}$. Camundongos SWISS com 35 dias de infecção pelo Schistosoma mansoni receberam tártaro emético por via intraperitoneal em dose única (10 $\mathrm{mg} \mathrm{Sb} / \mathrm{kg}$ ), seja na forma livre ou na forma encapsulada em lipossomas LC ou LF. Apenas o grupo tratado com LF mostrou redução significativa (55\%) no número de vermes, quando comparado

Tabela 4. Influência da sacarose nas características dos lipossomas DRVs e FDELs (composição lipídica: DSPC/DCP/CHOL em relação molar de 5:4:1) resuspendidos em solução salina tamponada $\left(\mathrm{NaCl} 0,15 \mathrm{~mol} \mathrm{~L}^{-1}\right.$; fosfato $\left.0,01 \mathrm{~mol} \mathrm{~L}^{-1} ; \mathrm{pH} 7,2\right)$

\begin{tabular}{lcccc}
\hline Tipo de lipossoma & $\begin{array}{c}\text { Relação inicial } \\
\text { Sb/lipídeo }(\mathrm{m} / \mathrm{m})\end{array}$ & $\begin{array}{c}\text { Diâmetro médio } \\
(\mathrm{nm}) \pm \mathrm{DP}\end{array}$ & $\begin{array}{c}\text { Taxa encapsulação } \\
(\% \mathrm{Sb}) \pm \mathrm{DP}\end{array}$ & $\begin{array}{c}\% \text { Sb liberado } \\
\text { após } 24 \mathrm{~h} \text { em } 37{ }^{\circ} \mathrm{C}\end{array}$ \\
\hline DRVs & 0,6 & $465 \pm 116$ & $61 \pm 3$ & $12,1 \pm 0,3$ \\
FDELs & 0,6 & $1205 \pm 451$ & $39 \pm 3$ & $3,7 \pm 0,4$ \\
DRVs + sacarose $^{\mathrm{a}}$ & 0,6 & $273 \pm 80$ & $42 \pm 14$ & $25,8 \pm 1,1$ \\
FDELs + sacarose $^{\mathrm{a}}$ & 0,6 & $312 \pm 182$ & $37 \pm 7$ & $24,1 \pm 3,1$ \\
\hline
\end{tabular}

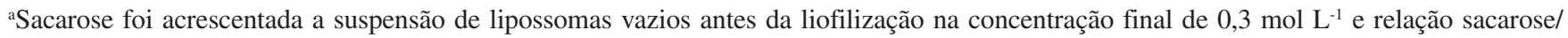
lipídeo 3:1 (m/m). DP: desvio padrão. 
aos grupos controles (grupo não tratado e grupo tratado com lipossomas vazios). A maior eficácia dos LF, quando comparados aos LC, pode ser atribuída ao aumento da biodisponibilidade do princípio ativo quando encapsulado em lipossomas furtivos, assim como à captura dos lipossomas de circulação prolongada pelos parasitas.

Quando submetidos a uma dose maior de antimônio (25 mg Sb/ $\mathrm{kg}$ ), todos camundongos (12/12) que receberam o tártaro emético na forma livre morreram, enquanto aqueles que receberam a forma encapsulada em lipossomas sobreviveram.

Conclui-se que os lipossomas furtivos reduzem a toxicidade aguda do tártaro emético e direcionam com eficácia a droga ao S. mansoni durante o curso da infecção. Esse estudo estabeleceu, pela primeira vez, o efeito benéfico dos vetores furtivos no tratamento da esquistossomose mansoni. Em uma outra abordagem, espera-se que a captura dos lipossomas pelo parasita possa ser aumentada através do acoplamento na superfície das vesículas de anticorpo ou ligante especifico para receptor/ligante do parasita.

\section{CONCLUSÕES}

A quimioterapia antimonial convencional sofre várias limitações, as mais sérias sendo a necessidade de injeções diárias, os seus efeitos colaterais e o aparecimento de resistência. As propriedades farmacológicas dos lipossomas "convencionais" fazem deste sistema um transportador ideal para direcionar medicamentos leishmanicidas para os sítios de infecção e aumentar sua eficácia no tratamento da leishmaniose visceral. No caso dos antimoniais pentavalentes, níveis de atividade espetaculares foram alcançados em modelo experimental de leishmaniose visceral, após sua administração na forma encapsulada em lipossomas. Entretanto, a obtenção de composição aceitável, do ponto de vista farmacológico e farmacêutico, continua sendo investigada. A literatura mostra que a taxa de encapsulação do antimonial pentavalente em lipossomas pode ser otimizada através da seleção do método de preparação e da composição lipídica da membrana. Por outro lado, a composição lipídica, o método de preparação e a pressão osmótica do compartimento interno são fatores determinantes para a obtenção de uma composição de lipossomas estável. Além disso, o tamanho das vesículas parece ser importante para atingir os locais de infecção menos acessíveis.

Recentemente, foi mostrado que a encapsulação de um antimonial trivalente em lipossomas reduz sua toxicidade aguda. Além disso, lipossomas "furtivos", ao contrário dos lipossomas "convencionais", mostraram-se capazes de aumentar a eficácia do antimonial trivalente em modelo experimental de esquistossomose mansoni. Esses resultados mostram que os lipossomas representam também sistemas de transporte promissores para os antimoniais trivalentes no tratamento das leishmanioses e da esquistossomose.

\section{AGRADECIMENTOS}

À Fundação de Amparo à Pesquisa do Estado de Minas Gerais (FAPEMIG) e ao Conselho Nacional de Desenvolvimento Científico e Tecnológico (CNPq) pela concessão de auxílios e bolsas.

\section{REFERENCIAS}

1. Vianna, G.; Resumos do $7^{\circ}$ Congresso Brasileiro de Medicina Tropical de São Paulo, Brasil, 1912.

2. Christopherson, J. B.; Lancet 1918, 2, 325.

3. Cioli, D.; Pica-Mattoccia, L.; Archer, S.; Pharmac. Ther. 1995, 68, 35.
4. Marsden, P. D.; Rev. Soc. Bras. Med. Trop. 1985, 18,187; Berman, J. D.; Clin. Infect. Dis. 1997, 24, 684; Rath, S.; Trivelin, L. A.; Imbrunito, T. R.; Tomazela, D. M.; de Jesús, M. N.; Marzal, P. C.; Junior H. F. A.; Tempone, A. G.; Quim. Nova 2003, 26, 550.

5. Goodwin, L. C.; Page, J. E.; Biochem. J. 1943, 22, 236.

6. Frézard, F.; Demicheli, C.; Ferreira, C. S.; Costa, M. A. P.; Antimicrob. Agents Chemother. 2001, 45, 913; Ferreira, C. S.; Martins, P. S.; Demicheli, C.; Brochu, C.; Ouellette, M.; Frézard, F.; BioMetals 2003, 16, 441.

7. Sturrock, R. F.; Mem. Inst. Oswaldo Cruz 2001, 96(Suppl.), 17.

8. Remme, J. H. F.; Blas, E.; Chitsulo, L.; Desjeux, P. M. P.; Engers, H. D.; Kanyok, T. R.; Kayondo, J. F. K.; Kioy, D. W.; Kumaraswami, V.; Lasdins, J. K.; Nunn, P. P.; Oduola, A.; Ridley, R. G.; Toure, Y. T.; Zicker, F.; Morel, C. M.; Trends Parasitol. 2002, 18, 421; Ridley, R. G. Em Drug against Parasitic Diseases; Failamb, A. H. ; Ridley, R. G. ; Vial, H. J., eds.; UNDP/ World Bank/WHO; Special Programme for Research and Training in Tropical Diseases (TDR); Geneva, 2003, p. 13.

9. Frézard, F.; Braz. J. Med. Biol. Res. 1999, 32, 181; Santos, N. C.; Castanho, M. A. R. B.; Quim. Nova 2002, 25, 1181.

10. Bangham, A. D.; Standish, M. M.; Watkins, J. C.; J. Mol. Biol. 1965, 13, 238.

11. New, R. R.; Chance, M. L.; Thomas, S. C.; Peters, W.; Nature 1978, 272, 55; Alving, C. R.; Steck, E. A.; Chapman, W. L.; Waits, V. B.; Hendricks, L. D.; Swartz, G. M.; Hanson W. L.; Proc. Natl. Acad. Sci. U.S.A. 1978, 75, 2959.

12. Frézard, F.; Santaella, C.; Vierling, P.; Riess, J. G.; Biochim. Biophys. Acta 1994, 1192, 61.

13. Crowe, L. M.; Crowe, J. H. Em Liposomes, New Systems and New Trends in Their Applications; Puisieux, F.; Couvreur, P.; Delattre, J.; Devissaguet, J. P., eds.; Editions de Santé: France, 1995, cap. 8.

14. Fattal, E.; Ramaldes, G. A.; Ollivon, M. Em ref. 13, cap. 24.

15. Woodle, M. C., Lasic, D. D.; Biochim. Biophys. Acta 1992, 1113,171.

16. Egbaria, K.; Weiner, N; Adv. Drug Deliv. Rev. 1990, 5, 287; Touitou, E.; Junginger, H. E.; Weiner, T.; Nagai, T.; Mezei, M.; J. Pharm. Sci. 1994, $83,1189$.

17. Neves, D. P.; Melo, A. L.; Genaro, O.; Linardi, P. M.; Parasitalogia Humana, 9a ed.; Atheneu: São Paulo, 1995, p. 212; D'arcy, P. F.; Harron, D. W. G.; Pharm. Int. 1983, 4, 16.

18. UNDP/World Bank/WHO Em Tropical Disease Research Programme Report, 13; progress 1995-96; World Health Organization: Geneva, 1997, cap. 8 .

19. http://portalweb02.saude.gov.br/saude, acessada em Agosto 2004.

20. Black, C. D. V.; Watson, G. J.; Ward, R. J.; Trans. Roy. Soc. Trop. Med. Hyg. 1977, 71, 550.

21. Collins, M., Carter, K. C., Baillie, A. J., O’Grady, J.; J. Drug Targeting 1993, 1,133 .

22. Schettini, D. A.; Costa Val, A. P.; Souza, L. F.; Demicheli, C.; Rocha, O. G. F.; Melo, M. N.; Michalick, M. S. M.; Frézard, F.; Braz. J. Med. Biol. Res. 2003, 36, 269.

23. Carter, K. C.; Dolan, T. F.; Alexander, J.; Baillie, A. J.; McColgan, C.; J. Pharm. Pharmacol. 1989, 41, 87.

24. Davidson, R. N.; di Martino, L.; Gradoni, L.; Giacchino, R.; Gaeta, G. B.; Pempinello, R.; Scotti, S.; Cascio, A.; Castagnola, E.; Maisto, A.; Gramiccia, M.; di Caprio, D.; Wilkinson, R. J.; Bryceson, A. D.; Clin. Infect. Dis. 1996, 22, 938; Meyerhoff, A.; Clin. Infect. Dis. 1999, 28, 42.

25. Alkhawajah, A. M.; Larbi, E.; al-Gindan, Y.; Abahussein, A.; Jain, S.; Ann. Trop. Med. Parasitol. 1997, 91, 899.

26. Alving, C. R.; Swartz, G. M. Em Liposome Technology; Gregoriadis, G., ed.; CRC Press: Boca Raton, 1984, vol. II, cap. 4.

27. Rao, L. S.; US pat. 4,594,241 1986.

28. Kirby, C.; Gregoriadis, G.; Biotechnology 1984, 2, 979.

29. Frézard, F.; Michalick, M. S. M.; Soares, C. F.; Demicheli, C.; Braz. J. Med. Biol. Res. 2000, 33, 841

30. Schettini, D. A.; Demicheli, C.; Rocha, O. G. F.; Frézard, F.; trabalho não publicado.

31. Zadi, B.; Gregoriadis, G.; J. Lipos. Res. 2000, 10, 73.

32. Frézard, F.; Melo, A. L.; Rev. Inst. trop. São Paulo 1997, 39, 97; El-Ridy, M. S.; Khalil, R. M.; Moustafa, D. M.; Mohamed, E.-R. M.; Osman, A.; Gaber, M. H.; Monem, A. S.; Drug Develop. Ind. Pharm. 1997, 23, 771.

33. Ammar, H. O.; El-Ridy, M. S.; Ghorab, M.; Ghorab, M. M.; Int. J. Pharm. 1994, 103, 237.

34. El-Ridy, M.; Akbarieh, M.; Kassem, M.; Sharkawi, M.; Tawashi, R.; Int. J. Pharm. 1989, 56, 23.

35. de Melo, A. L.; Silva-Barcellos, N. M.; Demicheli, C.; Frézard, F.; Int. J. Pharm. 2003, 255, 227. 